\section{Humeruskopffrakturen: Ergebnis nach winkelstabiler Plattenosteosynthese}

Schnetzke M et al. Quality of Reduction Influences Outcome After Locked-Plate Fixation of Proximal Humeral Type-C Fractures. J Bone Joint Surg Am 2016; 98: 1777 - 1785

Die proximale Humerusfraktur kann herausfordernd sein, insbesondere wenn eine begleitende Osteoporose oder multifragmentäre Fraktur vorliegt. Die Frakturen können meist konservativ behandelt werden. Bei jungen Patienten und instabilen, dislozierten Frakturen wird eine operative Versorgung empfohlen. Inwieweit die Qualität der Reposition der Fraktur das funktionelle Ergebnis nach winkelstabiler Plattenosteosynthese beeinflusst, ist noch nicht bekannt.

\section{Methoden}

Dislozierte Humeruskopffrakturen vom Typ C nach der OTA/AO-Klassifikation wurden mit einer winkelstabilen Plattenosteosynthese (Philos, Fa DePuy Synthes) opera- tiv versorgt. Als operativer Zugang diente in allen Fällen ein deltoideopektoraler Zugang. Im Rahmen dieser retrospektiven Analyse wurden die Patienten einer klinischen und radiologischen Nachuntersuchung unterzogen. Das funktionelle Ergebnis wurde gemessen anhand des Constant- und DASH-Scores. Zur Analyse des Repositionsergebnisses wurde eine Röntgenuntersuchung des Humeruskopfs in 2 Ebenen unmittelbar postoperativ herangezogen. Gemessen wurde der Versatz zwischen Kopf- und Schaftfragment, die Kranialisation des Tuberculum majus und die Angulation zwischen Kalotte und Schaft. Diese Messungen wurden mit den funktionellen Ergebnissen der Nachuntersuchung korreliert.

\section{Ergebnisse}

Nach durchschnittlich 3,1 $( \pm 1,5)$ Jahren konnten 98 Patienten mit einer komplexen Humeruskopffraktur zu einer Nachuntersuchung gesehen werden. Bei 40 Patienten konnte eine anatomische bzw. akzeptable Reposition erzielt werden. Eine qualitativ schlechte Reposition führte zu nachweisbar schlechteren funktionellen Ergebnissen. Insbesondere eine Kranialisation des Tuberculum majus oder eine SchaftKopf-Dislokation um mehr als $5 \mathrm{~mm}$ sowie eine starke Varus- oder Valgus-Fehlstellung $\left(<110^{\circ}\right.$ oder $\left.>150^{\circ}\right)$ konnten mit einem deutlich schlechteren funktionellen Ergebnis korreliert werden. Interessanterweise hatte das Alter der Patienten keinen Einfluss auf das Ergebnis.

\section{FAZIT}

Die Autoren schlussfolgern, dass die Qualität der Frakturreposition ein entscheidender Faktor für das funktionelle Ergebnis nach operativer Versorgung von Humeruskopffrakturen ist. Kritisch bleibt hier sicherlich anzumerken, dass auch nach konservativer Behandlung von Humeruskopffrakturen gute Ergebnisse berichtet werden und das, obwohl hier häufig keine anatomische Reposition erzielt werden kann. Darüber kann diese Untersuchung jedoch keine Aussage treffen. Wenn man die Entscheidung zur operativen Therapie stellt, sollte unter allen Umständen eine möglichst anatomische Reposition angestrebt werden, um für die betroffenen Patienten einen maximalen Funktionserhalt der Schulter zu erzielen.

Prof. Dr. Philip Gierer, Rostock 